(C) 2012 IEEE. Reprinted, with permission, from S. Krone and G. Fettweis, Capacity of Communications Channels with 1-Bit Quantization and Oversampling at the Receiver, in Proceedings of the IEEE Sarnoff Symposium (Sarnoff 12), Newark, USA, 21. - 22. Mai, 2012.

This material is posted here with permission of the IEEE. Such permission of the IEEE does not in any way imply IEEE endorsement of any of the products or services of Technical University Dresden. Internal or personal use of this material is permitted. However, permission to reprint/republish this material for advertising or promotional purposes or for creating new collective works for resale or redistribution must be obtained from the IEEE by writing to pubs-permissions@ieee.org. By choosing to view this document, you agree to all provisions of the copyright laws protecting it. 


\title{
Capacity of Communications Channels with 1-Bit Quantization and Oversampling at the Receiver
}

\author{
Stefan Krone and Gerhard Fettweis \\ Vodafone Chair Mobile Communications Systems \\ Technische Universität Dresden, 01062 Dresden, Germany \\ E-mail: $\{$ stefan.krone, fettweis\}@ifn.et.tu-dresden.de
}

\begin{abstract}
Communications receivers that rely on 1-bit analogto-digital conversion are advantageous in terms of hardware complexity and power dissipation. Performance limitations due to the 1-bit quantization can be tackled with oversampling. This paper considers the oversampling gain from an information-theoretic perspective by analyzing the channel capacity with 1-bit quantization and oversampling at the receiver for the particular case of AWGN channels. This includes a numerical computation of the capacity and optimal transmit symbol constellations, as well as the derivation of closed-form expressions for large oversampling ratios and for high signal-to-noise ratios of the channel.
\end{abstract}

\section{INTRODUCTION}

Communications receivers with multi-bit analog-to-digital converters (ADCs) require an analog gain control to optimally adjust the received signal amplitudes to the quantization grid of the ADCs [1]. This can be omitted when restricting to 1-bit ADCs, which can significantly reduce the complexity and power dissipation of communications receivers. The main drawback of 1-bit ADCs is, however, that the spectral efficiency of a complex-valued signal transmission will be limited to no more than 2 bits per channel use (bpcu) when sampling at symbol rate. The spectral efficiency can be improved through oversampling, which maintains the advantages of low complexity but can provide an effective quantization resolution larger than 1 bit. This paper discusses the prospects of 1-bit quantization and oversampling from an information-theoretic perspective, extending the authors' initial results published in [2]. It analyzes the capacity of additive white Gaussian noise (AWGN) channels with 1-bit quantization and oversampling at the receiver when the transmitted symbols assume rectangular pulse shapes without inter-symbol-interference. This is rather idealistic, but it allows to render the opportunities of 1-bit quantization and oversampling analytically, i.e., to describe the performance gain to be obtained with oversampling in general. The results are essential to understand and utilize the oversampling gain for more realistic channels, where the expected improvements build on the same general effects.

There is very few work that has discussed output quantization together with oversampling for digital communications channels. Most of the related work considers the overall statistics of the ADC input signals while neglecting that received communications signals are often composed of a useful signal part and unintended distortion caused by the transmission channel. The achievable rate of real-valued band-limited channels without noise but with 1-bit quantization and oversamp- ling at the receiver has been analyzed in [3]. For Gaussian distributed channel inputs it has been found that 2-fold oversampling (with respect to the channel bandwidth), can improve the achievable rate from $1 \mathrm{bpcu}$ to $1.072 \mathrm{bpcu}$, though it is argued that Gaussian distributed channel inputs may not be optimal to maximize the achievable rate. This conjecture has been pursued in [4], showing that 2-fold oversampling can yield up to 2 bpcu with properly designed channel inputs. For larger sampling rates, an upper bound of the achievable rate has been derived as $\xi \cdot \log _{2}(\xi)-(\xi-1) \cdot \log _{2}(\xi-1)$, where $\xi$ denotes the oversampling ratio (OSR). It is straight forward to extend this result to complex-valued channels without noise, which leads to twice this upper bound.

Only recently, the authors of [5] have started to analyze the capacity of AWGN channels with 1-bit quantization and oversampling at the receiver. The analysis has, however, been restricted to AWGN channels with very low signal-to-noise ratio (SNR), focusing on the capacity per unit cost, i.e., the ratio of the channel capacity and the average transmit power when the latter tends to zero. The results indicate that oversampling can improve the channel capacity at low SNR.

This paper extends the existing work by considering communications channels with AWGN, but without restricting to very high or very low SNRs only. The paper is organized as follows: The system model is explained in Section II. Section III reviews the authors' initial results [2], considering the achievable rate of AWGN channels with 1-bit output quantization and oversampling when conventional modulation schemes are used. The channel capacity and the capacity achieving transmit symbol constellations are calculated and discussed in Section IV. This includes an analytical derivation of the channel capacity at high SNR, and a derivation of a closed-form capacity expression for very large OSRs. Section V concludes the paper with a short summary and outlines topics for further research.

\section{SySTEM MODEL}

The system model under consideration is very similar to the model of direct sequence spread spectrum systems with 1-bit quantization at the receiver, which have been analyzed in [6]. It can be shown that a spreading sequence of $\xi$ ones corresponds to $\xi$-fold oversampling. This similarity will be used throughout the paper to adopt selected results of [6] for the analysis of the achievable rate and channel capacity with 1-bit quantization and oversampling at the receiver. 


\section{A. Discrete time system model}

Fig. 1 shows the discrete time model of an AWGN channel with 1-bit quantization and oversampling at the receiver, where $s=s_{\mathrm{I}}+j \cdot s_{\mathrm{Q}}$ denotes the complex-valued data symbols that are transmitted at symbol rate $f_{\text {symb. The respective received samp- }}$ les are denoted as $y[n]=y_{\mathrm{I}}[n]+j \cdot y_{\mathrm{Q}}[n]$. The model follows from the continuous-time representation of a complex-valued AWGN channel when two digital-to-analog converters (DACs) are used at the transmitter that operate at symbol rate and have a sufficiently large resolution to represent the in-phase $\left(s_{\mathrm{I}}\right)$ and quadrature-phase $\left(s_{\mathrm{Q}}\right)$ of the data symbols. The DAC output pulses are assumed to be rectangular, which reflects in the continuous-time impulse response $g_{\mathrm{DAC}}(t)=\sqrt{f_{\mathrm{symb}}} \cdot \operatorname{rect}\left(t \cdot f_{\mathrm{symb}}\right.$ $-0.5)$, where rect $(\cdot)$ denotes the rectangular function [7]. The factor $\sqrt{f_{\text {symb }}}$ scales the pulse shape to unit energy. The radio channel as well as the analog frontends of the transmitter and receiver have been assumed to be frequency flat, where $\theta$ denotes the overall channel attenuation, and $\phi$ is the channel phase. The power spectral density of the channel noise is denoted as $\mathcal{N}_{0}$. The sample-and-hold behavior of the two 1-bit ADCs at the receiver is taken into account with the continuous-time impulse response $g_{\mathrm{ADC}}(t)=\sqrt{\xi \cdot f_{\mathrm{symb}}} \cdot \operatorname{rect}\left(t \cdot \xi \cdot f_{\mathrm{symb}}-0.5\right)$. The ADC sampling rate is assumed to be an integer multiple of the symbol rate, i.e., $\xi \cdot f_{\text {symb }}$. With these assumptions, it is straight forward to show that the un-quantized received samples $x[n]=x_{\mathrm{I}}[n]+j \cdot x_{\mathrm{Q}}[n]$ of each transmitted symbol $s$ follow as

$$
x[n]=\frac{1}{\sqrt{\xi}} \cdot \theta \cdot e^{j \cdot \phi} \cdot s+w[n],
$$

where $n \in\{1,2, \ldots, \xi\}$. That is, each transmit symbol is represented by $\xi$ samples at the receiver without interference from earlier or later transmitted symbols. The factor $1 / \sqrt{\xi}$ results from the DAC and ADC impulse responses. It reflects the fact that a transmitted symbol is split into $\xi$ samples at the receiver while the total symbol energy is always the same, irrespective of $\xi$. The noise samples $w[n]=w_{\mathrm{I}}[n]+j \cdot w_{\mathrm{Q}}[n]$ are independent and identically Gaussian distributed with zero mean and variance $\sigma_{w}^{2}$, i.e., $w[n] \sim \mathrm{N}_{\mathbb{C}}\left(0, \sigma_{w}^{2}\right)$, where $\sigma_{w}^{2}=\mathcal{N}_{0}$. The quantized received samples $y[n]$ derive from $x[n]$ as

$$
y[n]= \begin{cases}q_{1}+j \cdot q_{1} & \text { for } \quad x_{\mathrm{I}}[n] \leq 0 ; x_{\mathrm{Q}}[n] \leq 0 \\ q_{1}+j \cdot q_{2} & \text { for } \quad x_{\mathrm{I}}[n] \leq 0 ; x_{\mathrm{Q}}[n]>0 \\ q_{2}+j \cdot q_{1} & \text { for } x_{\mathrm{I}}[n]>0 ; x_{\mathrm{Q}}[n] \leq 0 \\ q_{2}+j \cdot q_{2} & \text { otherwise }\end{cases}
$$

where $q_{1}$ and $q_{2}$ denote the two 1-bit quantization levels of the in-phase and quadrature-phase, respectively. The SNR of the AWGN channel is defined as the ratio of the average energy of a received symbol and $\mathcal{N}_{0}$. It is hence independent of $\xi$ and follows as

$$
\gamma=\theta^{2} \cdot \frac{\sigma_{s}^{2}}{\sigma_{w}^{2}}
$$

where $\sigma_{s}^{2}$ denotes the variance of the transmitted data symbols.

It might seem counterintuitive to exclude a low-pass receive filter that limits the received signal bandwidth to the symbol rate. The merit of this simplification is, however, that the noise samples $w[n]$ remain uncorrelated when $\xi>1$ while the recei-

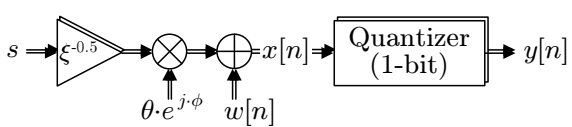

Fig. 1: Discrete time model of AWGN channels with 1-bit output quantization and $\xi$-fold oversampling.

ved symbol samples are fully correlated. This can improve the achievable rate and channel capacity when the quantization resolution at the receiver is as low as 1-bit, as shown in this paper.

\section{B. Transition Probabilities And Sufficient Statistic}

The system model given by (1) and (2) coresponds to a chanel with memory when $\xi>1$, since multiple received samples follow from each transmit symbol. By grouping the received samples in vectors of length $\xi$, where each vector contains the received samples of one transmit symbol, the channel can be transformed into an equivalent memoryless channel, where the output vectors are independent of each other. Let $\boldsymbol{y}$ denote the output vector of the received samples that belong to a transmit symbol. With the 1-bit quantization at the receiver, there are $\Lambda=4^{\xi}$ possible output vectors, which will be denoted as $\mathbf{y}_{\lambda}$ with $\lambda \in\left\{1,2, \ldots, 4^{\xi}\right\}$. The transition probabilities of the transformed memoryless channel follows as

$$
\operatorname{Pr}\left(\boldsymbol{y}=\boldsymbol{y}_{\lambda} \mid s=\mathrm{s}\right)=\prod_{n=1}^{\xi} \operatorname{Pr}\left([\boldsymbol{y}]_{n}=\left[\mathbf{y}_{\lambda}\right]_{n} \mid s=\mathbf{s}\right),
$$

where s denotes the possible channel inputs, i.e., the complexvalued transmit symbols. The factorization in (4) results from the fact that the AWGN samples are independent at all time instances. By introducing $\zeta_{\mathrm{I}}$ and $\zeta_{\mathrm{Q}}$ as

$$
\begin{gathered}
\zeta_{\mathrm{I}}=\Phi\left(\sqrt{2 \cdot \gamma} \cdot \mathfrak{R e}\left\{e^{j \cdot \phi} \cdot \mathrm{s}\right\} /\left(\sqrt{\xi} \cdot \sigma_{s}\right)\right) \\
\zeta_{\mathrm{Q}}=\Phi\left(\sqrt{2 \cdot \gamma} \cdot \mathfrak{I m}\left\{e^{j \cdot \phi} \cdot \mathrm{s}\right\} /\left(\sqrt{\xi} \cdot \sigma_{s}\right)\right),
\end{gathered}
$$

where $\Phi(\cdot)$ denotes the Gaussian cumulative distribution function [8], the factors in (4) can be written as follows:

$$
\begin{aligned}
& \operatorname{Pr}\left([\boldsymbol{y}]_{n}=\mathrm{y}_{1} \mid s=\mathrm{s}\right)=\zeta_{\mathrm{I}} \cdot \zeta_{\mathrm{Q}} \\
& \operatorname{Pr}\left([\boldsymbol{y}]_{n}=\mathrm{y}_{2} \mid s=\mathrm{s}\right)=\zeta_{\mathrm{I}} \cdot\left(1-\zeta_{\mathrm{Q}}\right) \\
& \operatorname{Pr}\left([\boldsymbol{y}]_{n}=\mathrm{y}_{3} \mid s=\mathrm{s}\right)=\left(1-\zeta_{\mathrm{I}}\right) \cdot \zeta_{\mathrm{Q}} \\
& \operatorname{Pr}\left([\boldsymbol{y}]_{n}=\mathrm{y}_{4} \mid s=\mathrm{s}\right)=\left(1-\zeta_{\mathrm{I}}\right) \cdot\left(1-\zeta_{\mathrm{Q}}\right) .
\end{aligned}
$$

Knowing the transition probabilities, it is possible to calculate the mutual information $\mathrm{I}(S ; \boldsymbol{Y})$ between the transmit symbols and the quantized received samples to analyze the achievable rate and the channel capacity. The calculation can be simplified by considering $q_{1}=0$ and $q_{2}=1$, without loss of generality, and introducing $y_{\Sigma}$ as the sum of the quantized received samples per transmit symbol:

$$
y_{\Sigma}=\sum_{n=1}^{\xi}[\boldsymbol{y}]_{n} \text {. }
$$

This sum is a sufficient statistic which reduces the cardinality of the possible channel outputs without affecting the mutual information, such that $\mathrm{I}\left(S ; Y_{\Sigma}\right)=\mathrm{I}(S ; \boldsymbol{Y})$. This can be shown by considering the data processing theorem [9], which implies $\mathrm{I}(S ; \boldsymbol{Y}) \geq \mathrm{I}\left(S ; Y_{\Sigma}\right)$, where equality holds if (and only if) $\boldsymbol{y}$ is independent of $\mathrm{s}$ when $y_{\Sigma}$ is known. The latter can be proven by verifying that the conditional probabilities $\operatorname{Pr}\left(\boldsymbol{y}=\mathbf{y}_{\lambda} \mid s=\mathrm{s}\right.$, $\left.y_{\Sigma}=\mathrm{y}_{\Sigma, \mathrm{I}}+j \cdot \mathrm{y}_{\Sigma, \mathrm{Q}}\right)$ are independent of $\mathrm{s}$. This is the case since 
$\operatorname{Pr}\left(\boldsymbol{y}=\mathbf{y}_{\lambda} \mid s=\mathrm{s}, y_{\Sigma}=\mathrm{y}_{\Sigma, \mathrm{I}}+j \cdot \mathrm{y}_{\Sigma, \mathrm{Q}}\right)=\left(\begin{array}{c}\xi \\ \mathrm{y}_{\Sigma, \mathrm{I}}\end{array}\right)^{-1} \cdot\left(\begin{array}{c}\xi \\ \mathrm{y}_{\Sigma, \mathrm{Q}}\end{array}\right)^{-1}$

where $\mathrm{y}_{\Sigma, \mathrm{I}}, \mathrm{y}_{\Sigma, \mathrm{Q}} \in\{0,1, \ldots, \xi\}$ denote the possible in-phase and quadrature-phase values that $y_{\Sigma}$ can assume, and (.) denotes the binomial coefficient [8]. (8) follows from the two facts that the noise samples $w[n]$ are independent and identically distributed and that all vectors $\boldsymbol{y}$ which yield the same $y_{\Sigma}$ are equally likely, irrespective of the particular transmit symbol s.

Note that (7) is a sufficient statistic for any channel phase $\phi$. The dependency on $\phi$ reflects in the probabilities of $y_{\Sigma}$. The sum in (7) transforms the original channel with $4^{\xi}$ possible output vectors into a memoryless channel with $(\xi+1)^{2}$ possible scalar outputs. The transition probabilities of the transformed channel derive from (4) by adding up all $\operatorname{Pr}\left(\boldsymbol{y}=\mathbf{y}_{\lambda} \mid s=\mathrm{s}\right)$ for which $\mathbf{y}_{\lambda}$ yields the same $y_{\Sigma}$ for a given s. This leads to

$\operatorname{Pr}\left(y_{\Sigma}=\mathrm{y}_{\Sigma, \mathrm{I}}+j \cdot \mathrm{y}_{\Sigma, \mathrm{Q}} \mid s=\mathrm{s}\right)=$

$\left(\begin{array}{c}\xi \\ y_{\Sigma, I}\end{array}\right) \cdot\left(1-\zeta_{\mathrm{I}}\right)^{\mathrm{y}_{\Sigma}, \mathrm{I}} \cdot \zeta_{\mathrm{I}}^{\left(\xi-y_{\Sigma, \mathrm{I}}\right)} \cdot\left(\begin{array}{c}\xi \\ \mathrm{y}_{\Sigma, \mathrm{Q}}\end{array}\right) \cdot\left(1-\zeta_{\mathrm{Q}}\right)^{\mathrm{y}_{\Sigma}, \mathrm{Q} \cdot \zeta_{\mathrm{Q}}}{ }^{\left(\xi-\mathrm{y}_{\Sigma, \mathrm{Q}}\right)}$,

which is the product of two binomial probability distributions $\mathrm{B}\left(\xi, \varsigma_{\mathrm{I}}\right)$ and $\mathrm{B}\left(\xi, \varsigma_{\mathrm{Q}}\right)[8]$, where $\varsigma_{\mathrm{I}}$ and $\varsigma_{\mathrm{Q}}$ are given in (5a-b).

\section{Performance With Conventional Modulation}

First observations of how oversampling can improve the performance of AWGN channels with 1-bit quantization at the receiver have been discussed in [2]. The performance has been evaluated in terms of the achievable rate when equally probable transmit symbols of an $L$-ary quadrature amplitude modulation ( $L$-QAM) scheme are used. The achievable rate is the mutual information between the transmitted $L$-QAM symbols and the respective quantized received samples. This is a lower bound of the channel capacity, which does already capture the main benefits of oversampling.

\section{A. General observations}

When the rectangular pulse of a received $L$-QAM symbol is sampled at multiple time instances, where each sample faces independent noise, it can happen that the different samples are (more ore less) randomly mapped to either quantization level due to the noise. This effect is more pronounced, the closer the amplitude of the received data symbol is to the quantization thresholds. The sum $y_{\Sigma}$ in (7) can then be used to resolve the location of the received data symbol in the complex plane from the vector of the quantized received samples. This allows to receive data symbols with an effective quantization resolution per in-phase and quadrature-phase that is larger than 1 bit. It relies, however, on the AWGN of the channel.

The respective results that have been discussed in [2] can be summarized as follows: Oversampling reduces the uncertainty of a received data symbol by observing multiple quantized samples, each of which is affected by independent noise. De- spite that, it also increases the uncertainty, because the energy of each sub-sample becomes smaller while the noise variance stays the same as compared to sampling at symbol rate. The latter dominates when 4-QAM symbols are transmitted over an AWGN channel with $\phi=0$, where oversampling causes a slight deterioration of the achievable rate. This is different if $\phi \neq 0$, where a strong degradation due to the channel phase can be mitigated through an improved effective quantization resolution that results with oversampling. This improvement can also be exploited to detect symbols of higher-order modulation schemes, such as 16-QAM. Considering 16-QAM, it has been observed that there is an optimal SNR below infinity which depends on $\phi$ and the OSR, where the achievable rate can attain a maximum of more than $2 \mathrm{bpcu}$, which would be the limit when sampling at symbol rate. This effect results from stochastic resonance [10]. However, the performance without quantization can hardly be achieved with a reasonably low OSR.

\section{B. Practical implications}

The effect that additive noise can improve the performance is closely related to dithered quantization. The main difference is, however, that the noise is not deliberately added but an inherent part of the transmission channel. If the noise bandwidth would be limited to the symbol rate, oversampling would not improve the performance, since the noise samples would be correlated. Hence, it is reasonable to scale the receiver bandwidth with the ADC sampling rate. When operating at high SNR, the lack of channel noise could be compensated with dedicated dithering. For very large OSRs, the optimal dither signal that maximizes the mutual information can be found in [10, pp. 150]. When the transmit symbol alphabet is discrete, the optimal dither signal will have the same number of discrete amplitudes. This is different from AWGN, which is hence not optimal. For reasonably low OSRs, the derivation of optimal dither signals still remains open.

Targeting at communications receivers with low complexity it may not be practical to include circuitry for dithered quantization, but rather to exploit the channel noise. By doing so, it is still possible to maximize the achievable rate, i.e., to achieve the channel capacity, with optimal transmit symbols, which is considered in the rest of the paper.

\section{Channel Capacity}

The channel capacity is defined as the maximum mutual information $\mathrm{I}\left(S ; Y_{\Sigma}\right)$ regarding all possible transmit symbol probability distributions. Allowing for arbitrary transmit symbol alphabets and taking an average transmit power constraint $\Omega$ into account, the channel capacity can be formulated as

$$
\mathrm{C}=\max _{\mathrm{p}_{s}(s)} \mathrm{I}\left(S ; Y_{\Sigma}\right) \quad \text { s.t. } \mathrm{E}\left\{|s|^{2}\right\} \leq \Omega / f_{\text {symb }},
$$

where $\mathrm{I}\left(S ; Y_{\Sigma}\right)$ is given in $(11)$, and $\mathrm{p}_{s}(s)$ denotes the probability density function of the transmit symbols. The integrals in

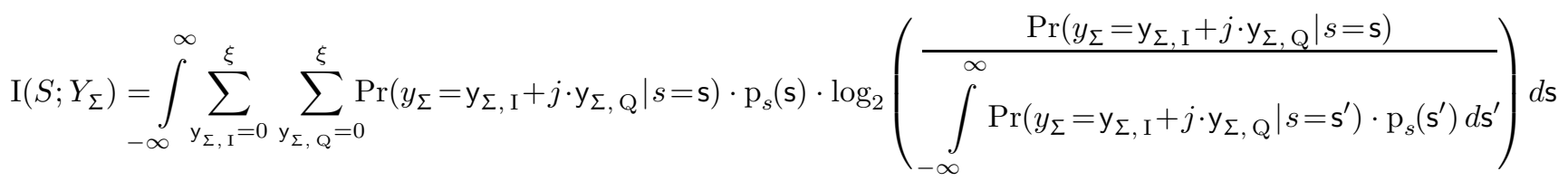


(11) are defined over the complex plane. It is difficult to derive a closed-form capacity expression that holds for the whole SNR range, as it is possible without oversampling [11]. Parts of the capacity investigations are therefore based on a numerical computation using the Cutting-Plane algorithm [12].

\section{A. Channel capacity at high SNR (analytical evaluation)}

The channel capacity at high SNR can be treated analytically. Following a similar derivation in [6], it can be shown that the capacity is achieved with transmit symbols taken from a 9QAM constellation. For the derivation, recall the transition probabilities given in (9), which follow with $\gamma \rightarrow \infty$ as $\left.\operatorname{Pr}\left(y_{\Sigma}=\mathrm{y}_{\Sigma, \mathrm{I}}+j \cdot \mathrm{y}_{\Sigma, \mathrm{Q}} \mid s=\mathrm{s}\right)\right|_{\gamma \rightarrow \infty}=$

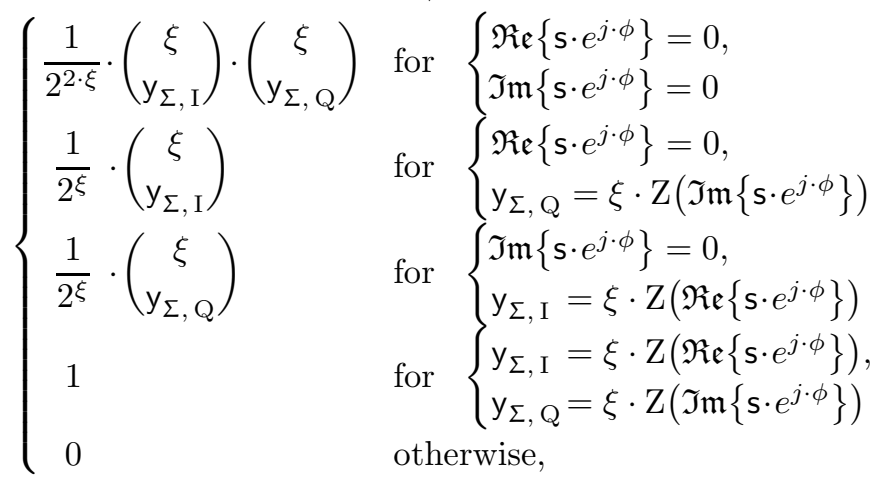

where $\mathrm{Z}(\cdot)$ denotes the step function [8] with $\mathrm{Z}(0)=0$. (12) results from the fact that the sub-samples of a received symbol at high SNR are mapped to either quantization bin with equal probability when the symbol falls on a quantization threshold. When the received symbol does not fall on a quantization threshold, all of its sub-samples are mapped to one quantization bin with probability 1 . Hence, the transition probabilities do not distinguish upon the exact value of a received symbol $s \cdot e^{j \cdot \phi}$, but only upon whether it falls in one of the quadrants of the complex plane or on either of the two quantization thresholds. This leads to 9 distinguishable symbols, for which the probability shall be defined as

$$
\mathrm{P}_{m_{\mathrm{I}}, m_{\mathrm{Q}}}=\operatorname{Pr}\left(\operatorname{sgn}\left(\mathfrak{R e}\left\{\mathbf{s} \cdot e^{j \cdot \phi}\right\}\right)=m_{\mathrm{I}}, \operatorname{sgn}\left(\mathfrak{R e}\left\{\mathbf{s} \cdot e^{j \cdot \phi}\right\}\right)=m_{\mathrm{Q}}\right)
$$

where $m_{\mathrm{I}}, m_{\mathrm{Q}} \in\{-1,0,1\}$, and $\operatorname{sgn}(\cdot)$ denotes the signfunction [8]. The maximum mutual information at high SNR is then given by (14), which requires a transmit symbol alphabet that provides all distinguishable symbols, e.g., 9-QAM. Another necessary condition to maximize $\mathrm{I}\left(S ; Y_{\Sigma}\right)$ is 4-fold rotational symmetry of the probability distribution of the transmitted symbols. This is straight-forward to show and requires

$$
\mathrm{P}_{m_{\mathrm{I}}, m_{\mathrm{Q}}}=\mathrm{P}_{-m_{\mathrm{I}}, m_{\mathrm{Q}}}=\mathrm{P}_{m_{\mathrm{I}},-m_{\mathrm{Q}}}=\mathrm{P}_{-m_{\mathrm{I}},-m_{\mathrm{Q}}},
$$

which implies that $\left.\mathrm{I}\left(S ; Y_{\Sigma}\right)\right|_{\gamma \rightarrow \infty}$ depends only on the values of $\mathrm{P}_{0,0}, \mathrm{P}_{1,0}$ and $\mathrm{P}_{1,1}$, i.e., on the probabilities that a received symbol falls in one of the quadrants, on either of the two quantization thresholds, or on both thresholds. Using this property and considering a similar derivation in [6], it can be shown that the capacity achieving transmit symbol probabilities at high SNR are given as a function of the OSR $\xi$ as follows:

$$
\mathrm{P}_{0,0}=\mathfrak{p}^{2} ; \quad \mathrm{P}_{0,1}=0.5 \cdot(1-\mathfrak{p}) \cdot \mathfrak{p} ; \mathrm{P}_{1,1}=0.25 \cdot(1-\mathfrak{p})^{2}
$$

with $\mathfrak{p}=\left(2 \cdot\left(2^{\xi \cdot 2^{\xi} /\left(2^{\xi}-2\right)}-1\right) / 2^{\xi}+1\right)^{-1}$. The parameter $\mathfrak{p}$ specifies every capacity achieving transmit probability distribution at high SNR by defining the probability that a received symbol falls on a quantization threshold. It leaves certain degrees of freedom for choosing a particular distribution. There is also no restriction on the average transmit power, i.e., every optimal transmit symbol alphabet can be scaled to satisfy any transmit power constraint while still achieving the same channel capacity at high SNR.

Fig. 2(a) shows the channel capacity at high SNR and the respective transmit symbol probabilities $\mathrm{P}_{0,0}$ as a function of the OSR. Note that $\mathrm{P}_{0,0}$ defines $\mathrm{P}_{1,0}$ and $\mathrm{P}_{1,1}$ through $\mathfrak{p}$. Fig. 2(b) shows examples of received symbol constellations that achieve the channel capacity at high SNR with a minimum number of constellation points. Without oversampling, an optimal transmit symbol alphabet has four constellation points that fall in the four quadrants of the complex domain with equal probability, where the channel capacity derives as $\mathrm{C}=2 \mathrm{bpcu}$. With oversampling, optimal transmit symbol alphabets have additional constellation points that fall on the quantization thresholds, where the probabilities are given by (16). When $\xi \rightarrow \infty$, the constellation points of the optimal transmit symbol alphabet fall in the quadrants and on the quantization thresholds with equal probability, where the channel capacity derives as $\mathrm{C}=\log _{2}(9)=3.699 \mathrm{bpcu}$. This is an upper limit which can be overcome only by reducing the SNR to exploit the effect of stochastic resonance.

\section{B. Channel capacity at arbitrary SNRs (numerical evaluation)}

The calculation of the channel capacity at arbitrary SNRs can be simplified by showing that it derives as the sum of the in-phase and quadrature-phase channel capacity when using each of the two real-valued channels independently with half the available transmit power and a phase offset correction. This implies that the capacity achieving received symbol constellation is always 4-fold rotational symmetric. The respective theorem and its proof are given in the appendix of this paper.

For the numerical capacity computation with the CuttingPlane algorithm, the in-phase and quadrature-phase of all possible transmit symbol distributions have been approximated with 401 uniformly spaced amplitude levels ranging from $-10 \cdot \sqrt{\Omega / f_{\text {symb }}}$ to $10 \cdot \sqrt{\Omega / f_{\text {symb }}}$. Fig. 3 shows the obtained results for different OSRs as a function of the SNR defined in

$$
\begin{array}{r}
\left.\mathrm{I}\left(S ; Y_{\Sigma}\right)\right|_{\gamma \rightarrow \infty}=\sum_{\mathrm{y}_{\Sigma, \mathrm{I}}=0}^{\xi} \sum_{\mathrm{y}_{\Sigma, \mathrm{I}}=0}^{\xi} \sum_{m_{\mathrm{I}}=-1}^{1} \sum_{m_{\mathrm{Q}}=-1}^{1} \mathrm{P}_{m_{\mathrm{I}}, m_{\mathrm{Q}}} \cdot \operatorname{Pr}\left(y_{\Sigma}=\mathrm{y}_{\Sigma, \mathrm{I}}+j \cdot \mathrm{y}_{\Sigma, \mathrm{Q}} \mid s=\left(m_{\mathrm{I}}+j \cdot m_{\mathrm{Q}}\right) \cdot e^{-j \cdot \phi}\right) \\
\cdot \log _{2}\left(\frac{\operatorname{Pr}\left(y_{\Sigma}=\mathrm{y}_{\Sigma, \mathrm{I}}+j \cdot \mathrm{y}_{\Sigma, \mathrm{Q}} \mid s=\left(m_{\mathrm{I}}+j \cdot m_{\mathrm{Q}}\right) \cdot e^{-j \cdot \phi}\right)}{\sum_{m_{\mathrm{I}}^{\prime}=-1}^{1} \sum_{m_{\mathrm{Q}}^{\prime}=-1}^{1} \mathrm{P}_{m_{\mathrm{I}}^{\prime}, m_{\mathrm{Q}}^{\prime}} \cdot \operatorname{Pr}\left(y_{\Sigma}=\mathrm{y}_{\Sigma, \mathrm{I}}+j \cdot \mathrm{y}_{\Sigma, \mathrm{Q}} \mid s=\left(m_{\mathrm{I}}^{\prime}+j \cdot m_{\mathrm{Q}}^{\prime}\right) \cdot e^{-j \cdot \phi}\right)}\right)
\end{array}
$$




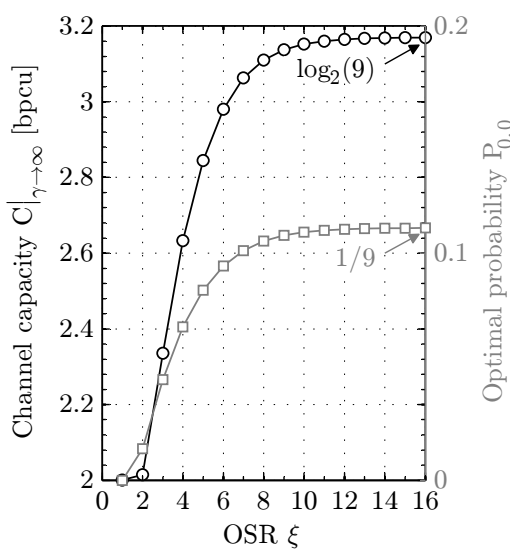

(a) Channel capacity $\mathrm{C}$ and optimal transmit symbol probability $\mathrm{P}_{0,0}$ versus OSR.
With oversampling $(\xi>1)$ :

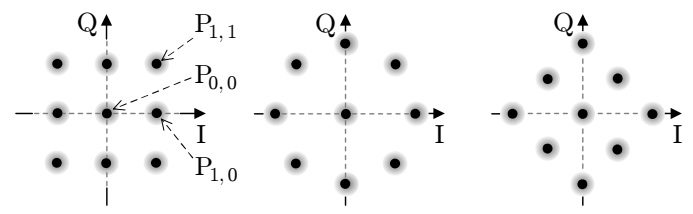

Without oversampling $(\xi=1)$ :

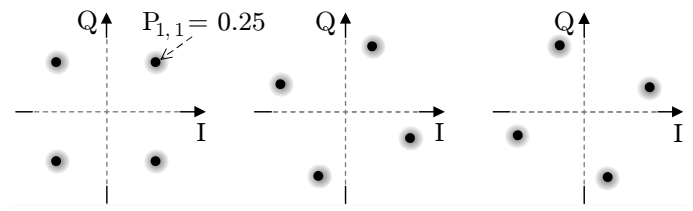

(b) Optimal received symbol constellations $s \cdot e^{j \cdot \phi}$ with the minimum number of constellation points (examples).

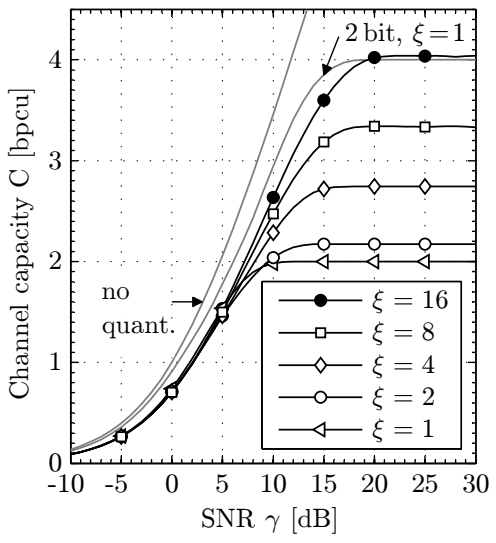

Fig. 3: AWGN channel capacity with 1-bit output quantization and $\xi$-fold oversampling: computed with the Cutting-Plane algorithm.

Fig. 2: Optimal usage of AWGN channels at high SNR with 1-bit output quantization and (no) oversampling.

(3) with $\sigma_{s}^{2}=\Omega / f_{\text {symb }}$. The capacity is compared to the channel capacity with 2-bit quantization when sampling at symbol rate, and to the capacity of an AWGN channel without quantization, which is independent of the OSR. It is obvious that oversampling significantly improves the channel capacity. Examples of the respective transmit symbol distributions $\mathrm{p}_{s}(s)=$ $\mathrm{p}_{s_{\mathrm{I}}, s_{\mathrm{Q}}}\left(s_{\mathrm{I}}, s_{\mathrm{Q}}\right)$, which have also been computed with the Cutting-Plane algorithm, are shown for 8-fold oversampling in Fig. 4. When $\phi \neq 0$, the optimal transmit symbol constellations are rotated by $e^{-j \cdot \phi}$, which compensates the channel phase. The transmit symbol distributions appear to have discrete mass points, where three SNR regions can be distinguished:

At very low SNR, where the AWGN determines the channel capacity, an optimal transmit symbol alphabet has four mass points with equal probability, irrespective of the OSR. This corresponds to a 4-QAM symbol constellation.

At high SNR, the small noise variance allows only to distinguish between symbols that are either close to or far away from the quantization thresholds. The optimal transmit symbol distribution converges to a 9-QAM symbol constellation with equally probable symbols when $\gamma \rightarrow \infty$.

The optimal transmit symbol distribution has a maximum number of mass points at medium SNR, where the noise variance is large enough to distinguish between more than 9 symbols. This is more pronounced the higher the OSR is. However, an analytical derivation of the optimal transmit symbol distribution as a function of the SNR and OSR remains open.

\section{Maximum channel capacity at high OSR (analytical eval.)}

Although it can barely be seen from the curves in Figure 3, the numerical results indicate that the channel capacity attains a maximum at an optimal SNR right after the turning points of the curves, e.g., at $\gamma \approx 20 \mathrm{~dB}$ for $\xi=8$. That is, the effect of stochastic resonance remains even if the transmit symbol distribution is optimized. The maximum channel capacity that results at an optimal SNR is shown as a function of the OSR in Figure 5. The curve has a logarithmic shape, but a closedform expression is again difficult to derive. Only for very large OSRs, it can be shown that the maximum channel capacity converges to

$$
\left.\max _{\gamma} \mathrm{C}\right|_{\xi \rightarrow \infty}=\log _{2}\left(\xi \cdot \frac{\pi}{2 \cdot e}\right) .
$$

This is twice the maximum capacity of the respective realvalued channel which has been considered in $[10$, Section 5.5]. The derivation of (17) builds on the fact that the transition probabilities given in (9) converge to a complex Gaussian probability distribution conditioned on $\mathrm{s}$ when the OSR tends to

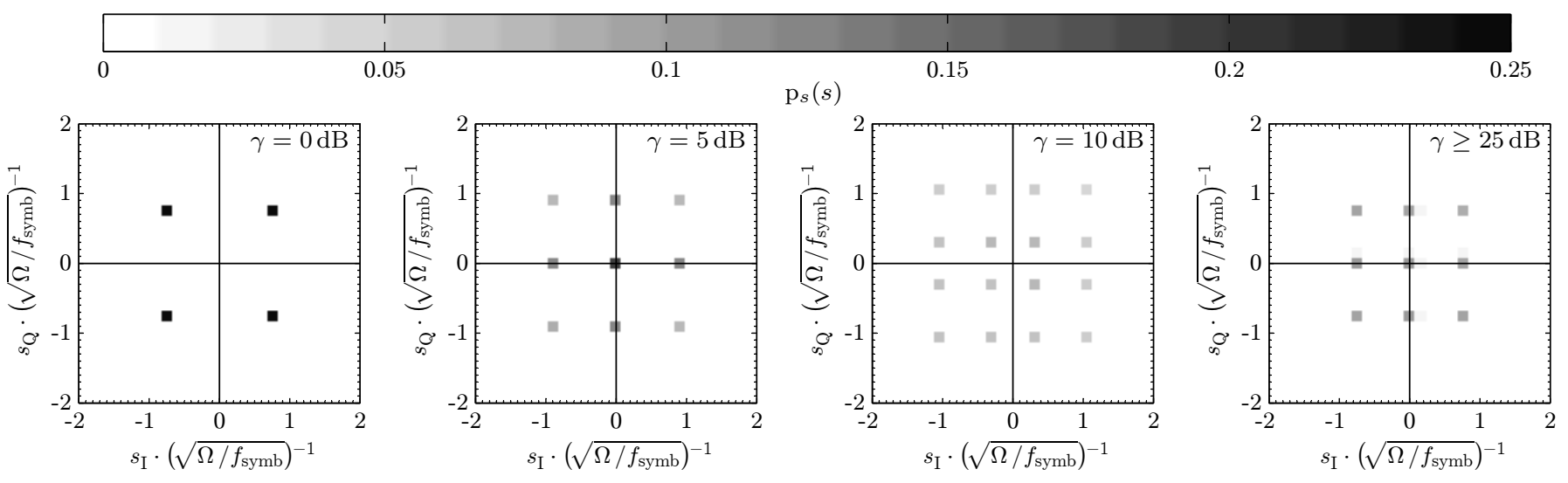

Fig. 4: Capacity-achieving transmit symbol distributions for AWGN channels with 1-bit quantization and 8-fold oversampling at the receiver: computed with the Cutting-Plane algorithm. The gray color shade indicates the probability values of the transmit symbol distribution. 


$$
\mathrm{p}_{s}(s)=\mathrm{p}_{s_{\mathrm{I}}, s_{\mathrm{Q}}}\left(s_{\mathrm{I}}, s_{\mathrm{Q}}\right)=\frac{e^{-\hat{\gamma} \cdot\left(s_{\mathrm{I}}^{2}+s_{\mathrm{Q}}^{2}\right) /\left(\xi \cdot \sigma_{s}^{2}\right)} \cdot \hat{\gamma} /\left(\xi \cdot \sigma_{s}^{2}\right)}{\pi^{3} \cdot \sqrt{\Phi\left(s_{\mathrm{I}} \cdot \sqrt{2 \cdot \hat{\gamma} /\left(\xi \cdot \sigma_{s}^{2}\right)}\right) \cdot \Phi\left(-s_{\mathrm{I}} \cdot \sqrt{2 \cdot \hat{\gamma} /\left(\xi \cdot \sigma_{s}^{2}\right)}\right) \cdot \Phi\left(s_{\mathrm{Q}} \cdot \sqrt{2 \cdot \hat{\gamma} /\left(\xi \cdot \sigma_{s}^{2}\right)}\right) \cdot \Phi\left(-s_{\mathrm{Q}} \cdot \sqrt{2 \cdot \hat{\gamma} /\left(\xi \cdot \sigma_{s}^{2}\right)}\right)}}
$$

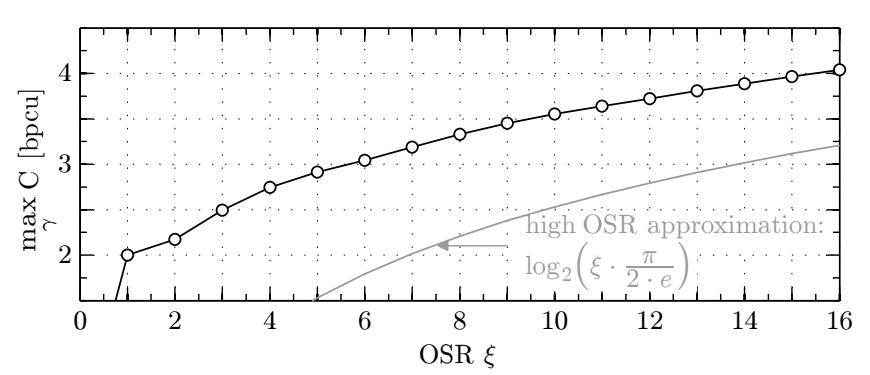

Fig. 5: Maximum capacity of AWGN channels with 1-bit quantization and $\xi$ fold oversampling at the receiver, which is attained at an optimal SNR that depends on the OSR $\xi$.

infinity, which follows from the central limit theorem [8]. The mean and the variance of this distribution are $\xi \cdot\left(\varsigma_{\mathrm{I}}+j \cdot \varsigma_{\mathrm{Q}}\right)$ and $\xi \cdot\left(\varsigma_{\mathrm{I}} \cdot\left(1-\varsigma_{\mathrm{I}}\right)+\varsigma_{\mathrm{Q}} \cdot\left(1-\varsigma_{\mathrm{Q}}\right)\right)$, where $\varsigma_{\mathrm{I}}$ and $\varsigma_{\mathrm{Q}}$ are given in (5a-b). Using this high SNR approximation of the transition probabilities and a separation of the channel into its in-phase and quadrature-phase parts, the remaining steps of the derivation are exactly the same as for the real-valued case considered in [10, pp. 150]. The transmit symbol distribution that yields the capacity maximum at very high OSR thus follows as given in (18), where $\sigma_{s}^{2}=\Omega / f_{\text {symb }}$, and $\hat{\gamma}$ denotes the optimal SNR that maximizes the channel capacity. $\hat{\gamma}$ increases linearly with the OSR and derives (numerically) from (18) as

$$
\hat{\gamma}=\xi \cdot \int_{-\infty}^{\infty} \int_{-\infty}^{\infty} \mathrm{p}_{s_{\mathrm{I}}, s_{\mathrm{Q}}}\left(s_{\mathrm{I}}, s_{\mathrm{Q}}\right) d s_{\mathrm{I}} d s_{\mathrm{Q}}=\xi \cdot 2.3266
$$

by setting $\hat{\gamma} /\left(\xi \cdot \sigma_{s}^{2}\right)=1$, without loss of generality. The transmit symbol distribution given in (18) requires a non-discrete transmit symbol alphabet and can be well approximated by a complex Gaussian distribution $\mathrm{N}_{\mathbb{C}}\left(0, \Omega / f_{\text {symb }}\right)$. However, Fig. 5 shows that (17) is not a convenient approximation of the maximum channel capacity for practical oversampling ratios. Nevertheless, it can still serve as a lower bound.

Finally, it should be stressed that the maximum channel capacity with oversampling is still less than the maximum entropy of $\xi$ random 1-bit quantized samples, i.e., less than $\xi \cdot 2$ bpcu. The reason is that the samples of each quantized received symbol are not independent. This is only case at very low SNR, where the channel capacity, however, tends to zero even without 1-bit quantization.

\section{CONCLUSIONS}

This paper has analyzed the capacity of communications channels with 1-bit quantization and oversampling at the receiver. The simple but still not trivial case of AWGN channels has been considered. The analysis has shown that oversampling can increase the channel capacity to much more than 2 bpcu. The oversampling gain requires a random distortion that acts like dithering at the quantizer inputs, which improves the effec- tive quantization resolution to more than 1 bit and thus allows to detect data symbols of high cardinality. The distortion should have a bandwidth that is larger than the symbol rate. The channel noise can be exploited for this purpose by adjusting the receiver bandwidth to the sampling rate. Examples of the capacity achieving transmit symbol constellations have been shown. One of the most interesting observations is that the channel capacity attains its maximum at an optimal SNR value that is below infinity, which results from stochastic resonance.

An alternative to utilizing the channel noise as random distortion could be to introduce dedicated inter-symbol-interference which may lead to similar improvements. This might be beneficial when operating at high SNR. Further research is required to identify the potential gains and optimal signal waveforms. Another open research topic is to extend the results of this paper to multi-bit quantization, which would have to include a fundamental investigation of the effect of stochastic resonance for quantization resolutions larger than 1-bit.

\section{ACKNOWLEDGMENTS}

This work has been supported in part by the German Research Foundation in the framework of the Collaborative Research Center 912 "Highly Adaptive Energy-Efficient Computing" and by the German Academic Exchange Service. The first author would like to thank Prof. U. Madhow (UC Santa Barbara) and Dr. J. Singh (Samsung Telecommunications America, Inc.) for the introduction to the Cutting-Plane algorithm.

\section{REFERENCES}

[1] S. Krone and G. Fettweis, "Optimal Gain Control for Single-Carrier Communications with Uniform Quantization at the Receiver," Proceedings of the IEEE International Conference on Acoustics, Speech and Signal Processing (ICASSP'10), 2010.

[2] _ "Achievable Rate with 1-Bit Quantization and Oversampling at the Receiver," IEEE Communication Theory Workshop (CTW'10), 2010.

[3] E. N. Gilbert, "Increased Information Rate by Oversampling," IEEE Transactions on Information Theory, vol. 39, no. 6, 1993.

[4] S. Shamai, "Information Rates by Oversampling the Sign of a Bandlimited Process," IEEE Trans. Information Theory, vol. 40, no. 4, 1994.

[5] T. Koch and A. Lapidoth, "Increased Capacity per Unit-Cost by Oversampling," IEEE Convention of Electrical and Electronics Engineers in Israel (IEEEI'10), 2010.

[6] O. Dabeer, J. Singh, and U. Madhow, "On the Limits of Communication Performance with One-bit A-D Conversion," IEEE Workshop on Signal Processing Advances in Wireless Communications (SPAWC'06), 2006.

[7] S. Haykin and B. van Veen, Signals and Systems. John Wiley \& Sons, Inc., 1998.

[8] I. N. Bronstein, K. A. Semendjajew, G. Musiol, and H. Mühlig, Handbook of Mathematics. Springer-Verlag, 2007.

[9] T. M. Cover and J. A. Thomas, Elements of Information Theory. John Wiley \& Sons, Inc., 1991.

[10] M. D. McDonnell, N. G. Stocks, C. E. M. Pearce, and D. Abbot, Stochastic Resonance - From Suprathreshold Stochastic Resonance to Stochastic Signal Quantization. Cambridge University Press, 2008.

[11] J. Singh, O. Dabeer, and U. Madhow, "Communication Limits with Low Precision Analog-to-Digital Conversion at the Receiver," Proceedings of the IEEE International Conference on Communications (ICC'07), 2007.

[12] J. Huang and S. P. Meyn, "Characterization and Computation of Optimal Distributions for Channel Coding," IEEE Transactions on Information Theory, vol. 51, no. 7, 2005.

[13] R. G. Gallager, Information Theory and Reliable Commuunication. John Wiley \& Sons, Inc., 1968. 


\section{APPENDIX}

Theorem 1. The capacity of complex-valued AWGN channels with a phase offset $\phi$, average transmit power constraint $\Omega$, integer oversampling and 1-bit quantization at the receiver can be achieved with channel input PDFs that satisfy

$$
\mathrm{p}_{s}(s)=\operatorname{p}_{\text {real }}\left(\mathfrak{R e}\left\{s \cdot e^{-j \cdot \phi}\right\}\right) \cdot \operatorname{p}_{\text {real }}\left(\mathfrak{I m}\left\{s \cdot e^{-j \cdot \phi}\right\}\right),
$$

where $\mathrm{p}_{\text {real }}(\cdot)$ denotes the channel input PDF that achieves the capacity $\mathrm{C}_{\text {real }}$ of either of the two real-valued channels (inphase and quadrature-phase) with an average transmit power constraint $\Omega_{\text {real }}=\Omega / 2$ when $\phi=0$. The capacity is

$$
\mathrm{C}=2 \cdot \mathrm{C}_{\text {real }} \text {. }
$$

Proof: The proof builds on a separation of the channel model into a front and a rear part, as depicted in Fig. 6. The front part accounts for the channel phase $\phi$, while the rear part captures the channel gain, the AWGN, the 1-bit quantization and the sum of the quantized samples per received symbol for the in-phase and quadrature-phase, respectively. The sum of the quantized received samples translates the oversampled section of the channel into a symbol-rate channel with scalar inputs and scalar outputs, without changing the mutual information (see Section II-B).

Let $\varpi=\varpi_{\mathrm{I}}+j \cdot \varpi_{\mathrm{Q}}$ denote the transmit symbols being affected by the channel phase, as shown in Fig. 6. These symbols have the same average power as the original transmit symbols and therefore satisfy the same constraint:

$$
\begin{aligned}
& \frac{\Omega}{f_{\text {symb }}} \geq \mathrm{E}_{s}\left\{|s|^{2}\right\}=\mathrm{E}_{\varpi}\left\{|\varpi|^{2}\right\} \\
& =\int_{-\infty-\infty}^{\infty} \int_{-\infty}^{\infty}\left(\varpi_{\mathrm{I}}^{2}+\varpi_{\mathrm{Q}}^{2}\right) \cdot \mathrm{p}_{\varpi_{\mathrm{I}}, \varpi_{\mathrm{Q}}}\left(\varpi_{\mathrm{I}}, u_{\mathrm{Q}}\right) d \varpi_{\mathrm{I}} d \varpi_{\mathrm{Q}} \\
& =\int_{-\infty}^{\infty} \varpi_{\mathrm{I}}^{2} \cdot \mathrm{p}_{\varpi_{\mathrm{I}}}\left(\varpi_{\mathrm{I}}\right) d \varpi_{\mathrm{I}}+\int_{-\infty}^{\infty} \varpi_{\mathrm{Q}}^{2} \cdot \mathrm{p}_{\varpi_{\mathrm{Q}}}\left(\varpi_{\mathrm{Q}}\right) d \varpi_{\mathrm{Q}}
\end{aligned}
$$

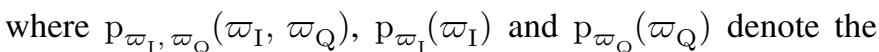
joint and marginal PDFs of the in-phase and quadraturephase components of $\varpi$. Now, consider the capacity $C^{\prime}$ of the rear part of the overall channel, i.e., the maximum mutual information between $\varpi=\varpi_{\mathrm{I}}+j \cdot \varpi_{\mathrm{Q}}$ and $y_{\Sigma}=y_{\Sigma, \mathrm{I}}+j \cdot y_{\Sigma, \mathrm{Q}}$ :

$$
\mathrm{C}^{\prime}=\max _{\mathrm{p}_{\varpi}(\varpi)} \mathrm{I}\left(\Pi ; Y_{\Sigma}\right) \quad \text { s.t. } \quad \mathrm{E}_{\varpi}\left\{|\varpi|^{2}\right\} \leq \Omega / f_{\text {symb }} .
$$

This capacity can be calculated by accounting for the fact that the rear part of the channel can be divided into two identical real-valued channels of the in-phase and quadrature-phase. The total capacity of these two parallel channels, each of which has capacity $\mathrm{C}_{\text {real }}$ under an average input power constraint $\Omega_{\text {real }}$, is $\mathrm{C}^{\prime}=2 \cdot \mathrm{C}_{\text {real }}$ under the total input power constraint $\Omega=$ $2 \cdot \Omega_{\text {real }}$. This follows from [13, Theorem 7.3.1] by taking into account that the two parallel channels can also be considered as a single channel which is sequentially used twice. The total capacity $\mathrm{C}^{\prime}$ is achieved by using each of the two parallel channels independently with the input PDF that achieves $\mathrm{C}_{\text {real }}$

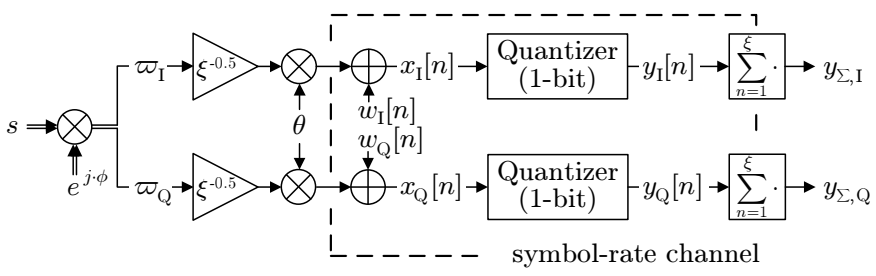

Fig. 6: Reformulated discrete time model of AWGN channels with 1-bit output quantization and oversampling.

under the input power constraint $\Omega_{\text {real }}$. The capacity achieving channel input PDF of the rear part of the complete channel can thus be factorized as

$$
\mathrm{p}_{\varpi}(\varpi)=\mathrm{p}_{\varpi_{\mathrm{I}}, \varpi_{\mathrm{Q}}}\left(\varpi_{\mathrm{I}}, \varpi_{\mathrm{Q}}\right)=\mathrm{p}_{\text {real }}\left(\varpi_{\mathrm{I}}\right) \cdot \mathrm{p}_{\text {real }}\left(\varpi_{\mathrm{Q}}\right) .
$$

It is straight forward to validate that the mutual information $\mathrm{I}\left(S ; Y_{\Sigma}\right)$ of the complete channel equals $\mathrm{I}\left(\Pi ; Y_{\Sigma}\right)$. Hence, it follows that $\mathrm{C}$ equals $\mathrm{C}^{\prime}$ and is achieved when $\mathrm{C}^{\prime}$ is achieved. The latter implies that the capacity-achieving input PDF of the complete channel has to be such that is satisfies (24), which is the case when (20) holds. This completes the proof. 